\title{
X-Ray Emission from Young Supernovae
}

\author{
Stefan Immler ${ }^{1}$ \\ Astronomy Department, University of Massachusetts, LGRT-B 528, 710 \\ North Pleasant Street, Amherst, MA 01003, USA
}

\begin{abstract}
Observations of young SNe in the ( 0.1-100 keV) X-ray band allow to establish physical key parameters, such as the circumstellar matter (CSM) density, the mass-loss rate of the progenitor and the temperature of the outgoing and reverse shock as a function of time. Over the last $\sim 25$ years, a small number of young SNe has been detected with orbiting X-ray observatories shortly after the outburst (days to months). Some highlights of recent observations with Chandra and XMM-Newton are presented and implications as to our understanding of the physical processes giving rise to the X-ray emission are briefly discussed.
\end{abstract}

\section{Introduction}

X-ray emission is expected from a core-collapsed SN shortly after its outburst ( hours-days) due to the interaction of the SN shock wave with the circumstellar material (CSM), which is present as a result of the progenitor's wind.

Different shock regions are formed as the ejecta plow into the CSM: the 'circumstellar' or 'forward shock', and the 'reverse shock' (see Chevalier \& Fransson 1994 and references therein). During the early phase after the outburst, the speed of the circumstellar shock is on the order of $\sim 10^{4} \mathrm{~km} \mathrm{~s}^{-1}$, while the speed of the reverse shock is $\sim 10^{3} \mathrm{~km} \mathrm{~s}^{-1}$ lower. Depending on the density profile of the ejecta, as they emerge from the star, the density behind the reverse shock can be 5-10 times higher than the density behind the circumstellar shock. The temperature behind the circumstellar shock can be as high as $10^{9-10} \mathrm{~K}$, whereas the temperature behind the reverse shock is significantly lower $\left(10^{7-8} \mathrm{~K}\right)$. Soft thermal X-ray $(\lessgtr 5 \mathrm{keV})$ emission is therefore produced by the reverse shock, whereas the higher energy X-rays $(z 10 \mathrm{keV})$ are more likely to arise in the forward shock region. Because of the much higher density in the reverse shock region, the $\mathrm{X}$-ray emission from this region will dominate that of the forward shock by the time the expanding shell has become optically thin enough to allow the X-rays from the reverse shock to leak out.

Over the last $\sim 25$ years, a small number of X-ray SNe has been detected, with X-ray luminosities in the range $10^{37}-10^{41} \operatorname{ergs~s}^{-1}$ (see Immler \& Lewin 2002 for a review article).

${ }^{1}$ current address: Department of Astronomy and Astrophysics, The Pennsylvania State University, 525 Davey Lab, University Park, PA 16802; email: immler@astro.psu.edu 


\section{X-Ray Production Mechanism}

The thermal X-ray luminosity, $L_{\mathrm{x}}$, produced by the shock heated CSM as the shock plows through, can be expressed as $L_{\mathrm{x}}=4 /\left(\pi m^{2}\right) \Lambda(T) \times\left(\dot{M} / v_{\mathrm{w}}\right)^{2} \times$ $\left(v_{\mathrm{s}} t\right)^{-1}$. Since the cooling function $\Lambda(T)$ is known for an observed temperature $T$, and the shock velocity $v_{\mathrm{s}}$ can be derived from radio observations, we can use the observed X-ray luminosity at time $t$ after the outburst to measure the ratio $\dot{M} / v_{\mathrm{w}}$. Each X-ray measurement at time $t$ is related to the corresponding distance $r$ from the site of the explosion. This site had been reached by the wind at a time depending on $v_{\mathrm{w}}$, or the age of the wind $t_{\mathrm{w}}=t v_{\mathrm{s}} / v_{\mathrm{w}}$. Usually, $v_{\mathrm{s}} \gg v_{\mathrm{w}}$ so that with $t$ only a few years we can look back quite a large time span in the evolution of the progenitor's wind and can use our measurements as a 'time machine' to probe the progenitor's history. Assuming that $v_{\mathrm{w}}$ did not change over $t_{\mathrm{w}}$, we can even directly measure the mass loss rate back in time. For a constant wind velocity $v_{\mathrm{w}}$ and mass-loss rate $\dot{M}$, a CSM density profile $\rho_{\mathrm{csm}}=\rho_{0}\left(r / r_{0}\right)^{-s}$ with $s=2$ is expected.

\section{3. $\mathrm{SN}$ 1993J in $\mathrm{M} 81$}

Analysis of the ROSAT X-ray lightcurve of SN 1993J, ranging from six days to five years after the outburst, were used to probe the progenitor's stellar wind history (Immler, Aschenbach \& Wang 2001). During the observed period, the SN shell has reached a radius of $3 \times 10^{17} \mathrm{~cm}$ from the site of the explosion, corresponding to $t_{\mathrm{w}} \sim 10^{4}$ years in the progenitor's stellar wind history. The ROSAT data showed that the mass-loss rate of the progenitor has decreased constantly from $\dot{M}=4 \times 10^{-4}$ to $4 \times 10^{-5} \mathrm{M}_{\odot} \mathrm{yr}^{-1}\left(v_{\mathrm{w}} / 10 \mathrm{~km} \mathrm{~s}^{-1}\right)$ during the late stage of the evolution. Assuming a spherically symmetric expansion, the CSM density profile was found to be significantly flatter $\left(\rho_{\operatorname{csm}} \propto r^{-1.63}\right.$; see Fig. 1) that what is expected for a constant mass-loss rate and constant wind velocity profile $\left(r^{-2}\right)$. This observed evolution was interpreted as either a decrease in the mass-loss rate, an increase in the wind speed or a combination of both. A likely explanation is that the progenitor was making a transition from the red to the blue supergiant phase during the late stage of its evolution, since blue supergiants are known to have faster and less dense stellar winds. This scenario for the evolution of the progenitor has interesting similarities with that of SN 1987A, whose progenitor completely entered the blue supergiant phase after mass-transfer to a companion.

\section{SN $1999 \mathrm{em}$ in NGC $\mathbf{1 6 3 7}$}

SN 1999em is the only Type IIP SN detected both in the radio and X-rays, although at a low flux level (day 4 after the outburst: $L_{\mathrm{x}}=7 \times 10^{37} \mathrm{ergs} \mathrm{s}^{-1}$ ). The Chandra X-ray data indicated a non-radiative interaction of the SN ejecta with the CSM and a low mass-loss rate of $\dot{M} \sim 2 \times 10^{-6} M_{\odot} \mathrm{yr}^{-1}$ (Pooley et al. 2002). Five Chandra observations, performed on days 4-368 after the outburst, showed a clear temperature evolution during this period, with a softening of the emission from originally $\sim 5 \mathrm{keV}$ to $\sim 1 \mathrm{keV}$ for an assumed thermal bremsstrahlung spectrum. This evolution confirmed predictions by the CSM in- 


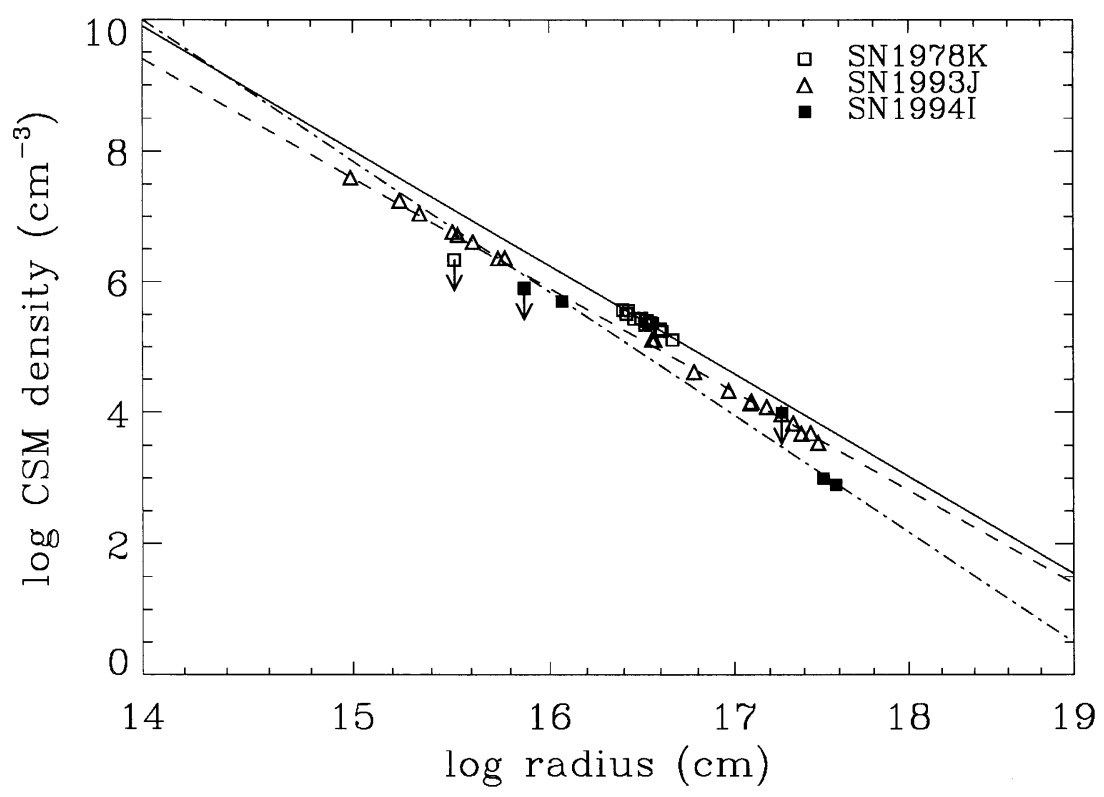

Figure 1. Circumstellar matter density profiles of SNe 1978K (open boxes, solid line) 1993J (triangles, dashed line) 1994I (filled boxes, dotted-dashed line), as a function of shell expansion radius.

teraction model of a change in temperature as the softer reverse shock emerges, and are indicative of a rather flat density profile of the ejecta.

\section{SN 1998 in NGC 3877}

A wealth of X-ray data has been collected for the Type IIn SN 1998S with Chandra and XMM-Newton. At an age of $\sim 2-3$ years after the outburst, SN 1998S is still bright in X-rays (Chandra data on day 668: $L_{\mathrm{x}} \sim 9 \times 10^{39} \mathrm{ergs} \mathrm{s}^{-1}$; Pooley et al. 2001; XMM-Newton data on day 1,202: $L_{\mathrm{x}} \sim 3 \times 10^{39} \mathrm{ergs} \mathrm{s}^{-1} ; 2-10 \mathrm{keV}$; this work) and increasing in $\mathrm{cm}$ radio flux density. The X-ray lightcurve based on five Chandra and one XMM-Newton observation is best described by an X-ray rate of decline of $L_{\mathrm{x}} \propto t^{-1.3}$. The inferred mass-loss rate is $\dot{M} \sim 2 \times 10^{-4} M_{\odot}$ $\mathrm{yr}^{-1}$. Spectral analysis of the Chandra data show a best-fit temperature of $\sim 10 \mathrm{keV}$ and high over-abundance of heavy elements, such as $\mathrm{Ne}, \mathrm{Al}, \mathrm{Si}, \mathrm{S}$, $\mathrm{Ar}$ and $\mathrm{Fe}$ (3-30 over solar). The high-quality spectra, in combination with theoretical modeling, allowed, for the first time, to use emission line ratios of elements produced before the core collapse and during the explosion to constrain the progenitor's mass $\left(\sim 18 M_{\odot}\right)$. A more recent XMM-Newton observation revealed the emergence of a soft $(\sim 0.8 \mathrm{keV})$ component from the reverse shock in addition to the harder component $(\sim 9 \mathrm{keV})$ already observed with Chandra. 


\section{6. $\mathrm{SN} 1994 \mathrm{I}$ in $\mathrm{M} 51$}

Recent high-resolution X-ray observations of the Type Ic SN 1994I with Chandra have conclusively shown, for the first time, that the interaction of the shock wave from a Type Ic SN with its ambient CSM can give rise to soft X-ray emission (Immler, Wilson \& Terashima 2002). Given an observed (0.3-2 keV band) Xray luminosity of $L_{\mathrm{x}} \sim 1 \times 10^{37} \mathrm{ergs} \mathrm{s}^{-1}$ between six and seven years after the outburst of SN 1994I, and assuming the X-ray emission arises from the shockheated CSM, a pre-SN mass-loss rate of $\dot{M} \sim 1 \times 10^{-5} M_{\odot} \mathrm{yr}^{-1}\left(v_{\mathrm{w}} / 10 \mathrm{~km} \mathrm{~s}^{-1}\right)$ was inferred. In combination with earlier ROSAT observations a best-fit X-ray rate of decline of $L_{\mathrm{x}} \propto t^{-s}$ with index $s \sim 1$ and a CSM density profile of $\rho_{\text {csm }} \propto r^{-1.9 \pm 0.1}$ were derived (see Fig. 1). The results are consistent with what is expected for a constant mass-loss rate and constant wind velocity profile for the SN progenitor $\left(\rho_{\mathrm{csm}} \propto r^{-2}\right)$.

\section{7. $\mathrm{SN} 1978 \mathrm{~K}$ in $\mathrm{NGC} \mathbf{1 3 1 3}$}

One of the best-studied SNe in the X-ray regime is SN 1978K. It has been successfully monitored with ROSAT on 13 different dates ranging from $\sim 12$ to 20 years after the outburst, as well as with subsequent $A S C A, C h a n d r a$ and XMMNewton observations. During this entire period, the SN showed no apparent evolution in X-rays $\left(L_{\mathrm{x}} \sim 4 \times 10^{39} \mathrm{ergs} \mathrm{s}^{-1}\right.$; Schlegel 1996, 1999). As expected for a Type II SN, a high and constant mass-loss rate of $\dot{M} \sim 1 \times 10^{-4} M_{\odot} \mathrm{yr}^{-1}$ was derived. A recent XMM-Newton observation showed that SN $1978 \mathrm{~K}$ is still at the same flux level as observed during the last $\sim 22$ years, showing no apparent evolution (Immler \& Aschenbach 2002). The superior sensitivity and spectral resolution of the XMM-Newton EPIC instruments (with a total of $\sim 20,000$ net counts) were used to constrain the spectral properties of SN 1978K. Similarly to SNe 1999em and 1998S, a significant 'softening' of the X-ray spectrum was found, with a best-fit two temperature model consisting of 'soft' $k T_{\text {low }} \sim 0.8 \mathrm{keV}$ component, absent in previous X-ray observations, and a harder $k T_{\text {high }} \sim 3 \mathrm{keV}$ component. The XMM-Newton data are interpreted as emission from the forward (high temperature component) as well as the reverse shock region (low temperature component), which was absent during earlier observations.

\section{References}

Chevalier, R.A., Fransson, C. 1994, ApJ, 420, 268

Immler, S., Lewin, W. 2002, in 'Supernovae and Gamma-Ray Bursts', ed. K.W. Weiler (Springer publisher), astro-ph/0202231

Immler, S., Wilson, A.S., Terashima, Y. 2002, ApJ, 573, L27

Immler, S., Aschenbach, B. 2002, in prep.

Immler, S., Aschenbach, B., Wang, Q.D. 2001, ApJ, 561, L107

Pooley, D., et al. 2002, ApJ, 572, 932

Schlegel, E.M., Petre, R., Colbert, J.M. 1996, ApJ, 456, 187

Schlegel, E.M. et al. 1999, AJ, 118, 2689 